\title{
EXCEEDANCE OF POWER BARRIERS FOR INTEGRATED CONTINUOUS-TIME STATIONARY ERGODIC STABLE PROCESSES
}

\author{
UĞUR TUNCAY ALPARSLAN, ${ }^{*}$ University of Nevada, Reno
}

\begin{abstract}
We study the asymptotic behavior of the tail probability of integrated stable processes exceeding power barriers. In the first part of the paper the limiting behavior of the integrals of stable processes generated by ergodic dissipative flows is established. In the second part an example with the integral of a stable process generated by a conservative flow is analyzed. Finally, the difference in the order of magnitude of the exceedance probability in the two cases is related to the dependence structure of the underlying stable process.
\end{abstract}

Keywords: Continuous-time stationary stable process; exceedance probability; nonlinear barriers; long-range dependence; integrated stable process

2000 Mathematics Subject Classification: Primary 60G52

Secondary 62P05

\section{Introduction}

Consider $S=\{S(t), t \geq 0\}$, a real-valued stochastic process, and a real-valued function $b(\cdot)$ on nonnegative reals representing a deterministic barrier. A classical problem in the theory of stochastic processes is to find the asymptotic behavior of the exceedance probability given by

$$
\psi(u)=\mathrm{P}\left[\sup _{t \geq 0}(S(t)-b(t))>u\right]
$$

as $u \rightarrow \infty$. The quantity $\psi(u)$ has several interpretations in different fields of applied probability. It is particularly important in insurance and queueing theory, and is also closely related to the tail probability of solutions to certain stochastic recurrence equations. There is a vast literature, specifically in the actuarial mathematics context, where the problem is treated under various assumptions. (For a list of references, see, for instance, [5].)

Classical models studying $\psi(u)$ (such as the Cramér-Lundberg model; see [11] for details), started off by assuming a process $S$, which had independent increments with finite exponential moments. However, empirical evidence in various fields, including finance, insurance, and teletraffic, supports the presence of heavy tails in real life. Moreover, in many of the application areas it is often more realistic to assume dependence among increments of $\boldsymbol{S}$. These observations have led to the recent efforts in the study of models where the increments of $\boldsymbol{S}$ are heavy tailed and dependent.

This scenario is also quite intriguing from a theoretical point of view as it brings up the possibility of gaining more insight into the dependence structure of stochastic processes with heavy tails by observing the asymptotic behavior of the tail probability of the functional $\psi$ on the sample paths. The problem becomes particularly interesting when the second moments

Received 8 December 2008; revision received 11 May 2009.

* Current address: Department of Mathematics and Statistics, American University, 4400 Massachusetts Avenue, NW, Washington, DC 20016, USA. Email address: tuncay.alparslan@american.edu 
cease to exist as the classical definitions of the range of dependence (which mainly rely on covariance like functions) become ambiguous.

In this paper we study the problem when $S$ is an integrated continuous-time process. Integrated processes are of interest on their own. In particular, integrated Brownian motion has been studied widely as it appears in classical literature in mechanics and biology (see, for instance, [12], [23], and [26], or [16]-[22] for a detailed account), as well as in quantum probability (see, for instance, [2] and [14]). Here, we will consider an integrated process described by

$$
S(t)=\int_{0}^{t} X(s) \mathrm{d} s, \quad t \geq 0,
$$

where $\boldsymbol{X}=\{X(t), t \geq 0\}$ is a stationary, ergodic, symmetric $\alpha$-stable ( $\mathrm{S} \alpha \mathrm{S}$ ) process with $\alpha \in(1,2)$. For this choice of $\alpha, X$ has a finite first moment; however, the second moment does not exist. This together with the fact that the structure of stable processes are relatively well understood allows us to concentrate on the underlying dependence structure in the presence of heavy tails. Furthermore, results for stable increments indicate to some extent what can be expected for more general processes, including stationary infinitely divisible processes. We believe the stable case will provide a benchmark which will help one to gain more insight into exceedances for more general processes.

Recently, there has been some effort in studying the asymptotics of $\psi(u)$ in the S $\alpha \mathrm{S}$ setting. Braverman [8] established an asymptotic equivalence between a class of subadditive functionals, including $\psi(u)$ given in (1), and a deterministic functional, which is based on the integral representation of the underlying stable process. However, explicit results on the order of magnitude of $\psi(u)$ were not the focus of that study. Mikosch and Samorodnitsky [24] gave results on the order of magnitude of the exceedance probabilities for discrete-time random walks driven by different classes of stationary ergodic $\mathrm{S} \alpha \mathrm{S}$ processes. Alparslan and Samorodnitsky [3], [4] extended some of the discrete-time results given in [24] and also established the order of magnitude for certain classes of integrated continuous-time processes. All of these studies, however, assumed a linear barrier.

The goal of this paper is to extend the results to exceedances of nonlinear barriers. In particular, we analyze the asymptotic behavior of $\psi(u)$ for power barriers of the form

$$
b(t)=\mu t^{p}, \quad t \geq 0, \quad \text { where } \mu>0 \text { and } p>1 .
$$

To analyze $\psi(u)$ for random walks driven by general classes of heavy-tailed, dependent processes, we need large deviation results which are not straightforward. In the case of stable processes, however, we make use of the representation of the stationary process with respect to a common stable random measure to obtain the tail behavior of the random walk.

Consider a measurable, stationary, ergodic $\mathrm{S} \alpha \mathrm{S}$ process $\boldsymbol{X}$ with $1<\alpha<2$. Without loss of generality, we will assume that

$$
X(t)=\int_{E} f_{t}(x) M(\mathrm{~d} x), \quad t \geq 0,
$$

where $M$ is an independently scattered $\mathrm{S} \alpha \mathrm{S}$ random measure on a measurable space $(E, \mathfrak{E})$ with a $\sigma$-finite control measure $m$ on $\mathfrak{E}$, and $\left\{f_{t}, t \geq 0\right\}$ is a subset of $L^{\alpha}(E, \mathfrak{E}, m)$. (See [30, Theorem 3.5.6].) Moreover, owing to the stationarity of the process, for $t \geq 0$, we can choose the kernel $f_{t}$ to be of a more depictive form given by

$$
f_{t}(\cdot)=a_{t}(\cdot)\left[\frac{\mathrm{d}}{\mathrm{d} m} m \circ \phi_{t}(\cdot)\right]^{\alpha} f \circ \phi_{t}(\cdot) .
$$


Here, $f$ is a real-valued function in $L^{\alpha}(E, \mathfrak{E}, m) ;\left\{\phi_{t}(\cdot), t \geq 0\right\}$ is a nonsingular flow on $E$; and $\left\{a_{t}, t \geq 0\right\}$ is a cocycle of the flow taking values on the unit circle. (For further details, see [27].) This representation allows us to relate certain path properties of a single kernel in $L^{\alpha}(E, \mathfrak{E}, m)$ and ergodic properties of a flow to the probabilistic structure of $\boldsymbol{X}$.

Indeed, one of the main objectives of this paper is to investigate the effects of an important result from infinite ergodic theory on the asymptotic behavior of $\psi(u)$ : Hopf decomposition of the flow implies that a stationary $\mathrm{S} \alpha \mathrm{S}$ process $\boldsymbol{X}$ can be represented in distribution as the sum of two independent stationary $\mathrm{S} \alpha \mathrm{S}$ processes $\boldsymbol{D}$ and $\boldsymbol{C}$, where the process $\boldsymbol{D}$ has an integral representation with the kernel given by (4) with a dissipative flow $\left\{\phi_{t}(\cdot), t \geq 0\right\}$, whereas the process $C$ has an integral representation with the kernel given by (4) with a conservative flow $\left\{\phi_{t}(\cdot), t \geq 0\right\}$. (For an in-depth discussion of dissipative and conservative flows, and Hopf decomposition, see, for instance, [1] or [15].) Rosiński [27] showed that any stationary $\mathrm{S} \alpha \mathrm{S}$ processes $\boldsymbol{D}=\left\{D(t), t \in \mathbb{R}_{+}\right\}$generated by a dissipative flow are automatically ergodic. Moreover, if the dissipative flow itself is ergodic then the process can be represented in distribution as a moving average

$$
\boldsymbol{D} \stackrel{\mathrm{D}}{=}\left\{\int_{\mathbb{R}} f(x-t) M(\mathrm{~d} x), t \in \mathbb{R}_{+}\right\}
$$

with $f \in L^{\alpha}(\mathbb{R}, \mathfrak{B}, \lambda)$, where $\mathfrak{B}$ is the Borel $\sigma$-algebra, $\lambda$ is the Lebesgue measure, $M$ is an $\mathrm{S} \alpha \mathrm{S}$ random measure, and ' $\stackrel{\mathrm{D}}{=}$ ' denotes equality in distribution.

This representation considerably simplifies the analysis of exceedance probabilities for random walks driven by dissipative stable processes. In Section 2 we will focus on this representation while studying the asymptotic behavior of $\psi(u)$ as $u \rightarrow \infty$. In this section we will also verify that the properties of $f$ can be significant in the determination of the order of magnitude of $\psi(u)$.

An analysis of exceedances for general stationary $\mathrm{S} \alpha \mathrm{S}$ processes generated by conservative flows is substantially harder. Far less is known about the structure of conservative stable processes compared to dissipative processes. Furthermore, even the construction of examples of such processes is not straightforward. In Section 3 we will concentrate on a certain class of conservative stable processes and show that $\psi(u)$ may decay slower than it does in the dissipative case, even with 'nice' kernels in the integral representation.

In Section 4 we will compare the main results of Section 2 and Section 3, and we will relate them to the dependence structure of the underlying stationary ergodic $\mathrm{S} \alpha \mathrm{S}$ process $\boldsymbol{X}$. Finally, proofs of these results will be presented in Section 5.

\section{Exceedances for stationary stable processes generated by ergodic dissipative flows}

Consider a stationary symmetric $\alpha$-stable process $\boldsymbol{X}=\{X(t), t \geq 0\}$ with $1<\alpha<2$, which is generated by an ergodic dissipative flow. As discussed in (5), these processes have a moving average representation; so, without loss of generality, assume that

$$
X(t)=\int_{\mathbb{R}} f(x-t) M(\mathrm{~d} x), \quad t \geq 0,
$$

where $f \in L^{\alpha}(\mathbb{R}, \mathfrak{B}, \lambda)$ and $M$ is an $\mathrm{S} \alpha \mathrm{S}$ random measure on $(\mathbb{R}, \mathfrak{B})$, with Lebesgue control measure $\lambda$. It follows from [30, Theorem 11.3.2] that the process $S=\{S(t), t \geq 0\}$ given by

$$
S(t)=\int_{0}^{t} X(s) \mathrm{d} s, \quad t \geq 0,
$$


is well defined. Moreover, defining $h_{t}(x)=\int_{0}^{t} f(x-s) \mathrm{d} s, t \geq 0$, we have, by [30, Theorem 11.4.1], $S(t)=\int_{\mathbb{R}} h_{t}(x) M(\mathrm{~d} x), t \geq 0$.

Let $b(\cdot)$ be a power barrier, as given in (2). Then, for $u>0$, the probability of exceeding the barrier by $u>0$ is given by

$$
\psi(u)=\mathrm{P}\left(\sup _{t \geq 0}\left(S(t)-\mu t^{p}\right)>u\right), \quad u>0 .
$$

Next, define

$$
\psi_{0}(u):=\frac{C_{\alpha}}{2} \int_{\mathbb{R}}\left(\sup _{t \geq 0} \frac{\left(h_{t}(x)\right)_{+}^{\alpha}}{\left(u+\mu t^{p}\right)^{\alpha}}+\sup _{t \geq 0} \frac{\left(-h_{t}(x)\right)_{+}^{\alpha}}{\left(u+\mu t^{p}\right)^{\alpha}}\right) \mathrm{d} x, \quad u>0,
$$

where

$$
C_{\alpha}=\left(\int_{0}^{\infty} x^{-\alpha} \sin x \mathrm{~d} x\right)^{-1}
$$

The importance of the functional $\psi_{0}$ arises from the following observation.

Proposition 1. As $u \rightarrow \infty, \psi(u) \sim \psi_{0}(u)$ if, for some $\gamma \in(0,1)$, the scaling parameter of $S(t)$ is $O\left(t^{\gamma}\right)$ as $t$ tends to $\infty$, i.e. if

$$
\left\|h_{t}(\cdot)\right\|_{L^{\alpha}(\mathbb{R}, \mathfrak{B}, \lambda)}=O\left(t^{\gamma}\right) \quad \text { as } t \rightarrow \infty
$$

This proposition is utilized in the proof of the theorem below, which is our main result for this section and shows the interplay between properties of the kernel $f$ in the moving average (6) and the asymptotic behavior of the exceedance probability (8).

Theorem 1. Let $f$ given in (6) be a nonnegative function, and suppose that there exist two integers, $K_{l}<0<K_{r}$, such that the function defined by

$$
g(x):=\sup _{t \geq 0} f(x-t) \mathbf{1}_{\left\{x \leq K_{l}\right\}}+f(x) \mathbf{1}_{\left\{K_{l}<x<K_{r}\right\}}+\sup _{t \geq 0} f(x+t) \mathbf{1}_{\left\{x \geq K_{r}\right\}}
$$

is in $L^{1}(\mathbb{R}, \mathfrak{B}, \lambda)$, and, for $x \geq K_{r}, g$ is bounded by a monotone, regularly varying function with index $-q<-1$ (i.e. there exists a monotone function $g_{\mathrm{rv}} \in R V_{-q}$ such that $g \leq g_{\mathrm{rv}}$ on $x \geq K_{r}$ ). Then, as $u \rightarrow \infty$,

$$
\psi(u) \sim \frac{C_{\alpha}}{2 p \mu^{1 / p}}\|f\|_{L^{1}(\mathbb{R}, \mathfrak{B}, \lambda)}^{\alpha} \mathcal{B}\left(\frac{1}{p}, \alpha-\frac{1}{p}\right) u^{1 / p-\alpha},
$$

where $\mathcal{B}(\cdot, \cdot)$ is the beta function.

\section{Exceedances for a class of stationary ergodic stable processes generated by conservative flows}

In this section we focus on a class of continuous-time random walks, where the increment process $\boldsymbol{X}$ is a continuous-time, stationary, ergodic $\mathrm{S} \alpha \mathrm{S}$ process generated by a conservative flow. The construction of such an $\boldsymbol{X}$ is due to Samorodnitsky [29], who used a standard fractional Brownian motion with self-similarity exponent $H \in(0,1)$ to construct the random measure $M(\cdot)$ given in the integral representation (3). Here we restrict our attention to the standard Brownian motion case (i.e. $H=0.5$ ), and we pick a fairly simple kernel for the 
integral representation of $\boldsymbol{X}$. In doing so we intend to show that even for this simple case with a 'nice' kernel, the order of magnitude of the exceedance probability is higher for the processes generated by conservative flows than that for the processes generated by dissipative flows.

Now let $\boldsymbol{B}=\{B(t), t \in \mathbb{R}\}$ be a standard Brownian motion. Let $m$ be a $\sigma$-finite cylindrical measure on $C(\mathbb{R})$, the space of continuous functions on $\mathbb{R}$, defined by

$$
m(A)=\int_{\mathbb{R}} \mathrm{P}(\boldsymbol{B} \in A-y) \mathrm{d} y, \quad \text { where } A \text { is a cylindrical set, }
$$

i.e. $m$ is the (infinite) law of the Brownian motion shifted according to the Lebesgue measure on $\mathbb{R}$. Define

$$
\varphi(x):=(1-|x|) \mathbf{1}_{\{(1-|x|) \in[0,1]\}}, \quad x \in \mathbb{R} .
$$

Note that $\varphi: \mathbb{R} \mapsto[0, \infty)$ is Hölder continuous with exponent 1 , even, and nonincreasing on $[0, \infty)$. Also, note that $\varphi \in L^{\alpha}(\mathbb{R}, \mathcal{B}, \lambda)$. Clearly, the Hölder function

$$
H(x)=\sup _{x \leq s<t} \frac{\varphi(s)-\varphi(t)}{t-s}, \quad x \geq 0,
$$

also belongs to $L^{\alpha}(\mathbb{R}, \mathcal{B}, \lambda)$. Furthermore, define

$$
X(t)=\int_{C(\mathbb{R})} \varphi\left(x_{t}\right) M(\mathrm{~d} \boldsymbol{x}), \quad t \in \mathbb{R}, \boldsymbol{x}=\left(x_{s}, s \in \mathbb{R}\right),
$$

where $M$ is an $\mathrm{S} \alpha \mathrm{S}$ random measure on $C(\mathbb{R})$ with control measure $m$. It was shown in [29] that the process $X=\{X(t), t \in \mathbb{R}\}$ is a well-defined stationary $\mathrm{S} \alpha \mathrm{S}$ process, and is generated by a conservative flow.

For $t \geq 0$, let $S(t)$ be as given in (7) and define

$$
h_{t}(\boldsymbol{x}):=\int_{0}^{t} \varphi\left(x_{s}\right) \mathrm{d} s .
$$

It was shown in [4] that the process $S:=\{S(t), t \geq 0\}$ is well defined, and, moreover,

$$
S(t)=\int_{C(\mathbb{R})} h_{t}(\boldsymbol{x}) M(\mathrm{~d} \boldsymbol{x}) \quad \text { almost surely (a.s.), } t \geq 0 .
$$

Let $\{L(x, t), x \in \mathbb{R}, t \geq 0\}$ be a jointly continuous local-time process of $\boldsymbol{B}$ defined by

$$
\int_{0}^{t} g[B(s)] \mathrm{d} s=\int_{\mathbb{R}} g(x) L(x, t) \mathrm{d} x
$$

for every bounded continuous function $g$ on $\mathbb{R}$. (See [6] for details.) By the self-similarity of $\boldsymbol{B}$, the local-time process has the following scaling property:

$$
\left\{L\left(c^{1 / 2} x, c t\right), x \in \mathbb{R}, t \geq 0\right\} \stackrel{\mathrm{D}}{=}\left\{c^{1 / 2} L(x, t), x \in \mathbb{R}, t \geq 0\right\}
$$

for any $c>0$. Moreover, all moments of $l(x, t)$ are finite, and are uniformly bounded in all real $x$ and all real $t$ in a compact set. (See, for instance, [9] for details.)

Let $\psi(u), u>0$, be as in (1) with $S(\cdot)$ given by (11). Now we state the main result of this section.

Theorem 2. In the setting described above, as $u \rightarrow \infty$,

$$
\psi(u) \sim \frac{C_{\alpha} \mathcal{B}(1 / 2 p,(2 p \alpha-\alpha-1) / 2 p)}{\mu^{(\alpha+1) / 2 p} p \sqrt{2 \pi}} \mathrm{E}\left[\left(\sup _{t \geq 0} \frac{L\left(0, t^{1 / p}\right)}{1+t}\right)^{\alpha}\right] u^{-(2 p \alpha-\alpha-1) / 2 p},
$$

where $\mathcal{B}(\cdot, \cdot)$ is the beta function. 


\section{Discussion}

In Section 2 we considered the case of stationary symmetric stable processes generated by ergodic dissipative flows. It was shown through evaluation of the exact limit that, under certain technical conditions imposed on the kernel in the integral representation,

$$
\psi(u)=O\left(u^{1 / p-\alpha}\right) \quad \text { as } u \rightarrow \infty .
$$

It should be noted that any function with compact support, or exponentially decaying tails, or tails decaying according to a power law with power smaller than -1 , satisfy the conditions imposed on the kernel.

In the case of stationary symmetric stable processes generated by ergodic conservative flows, however, even the processes with 'nice' kernels might result in a slower decaying exceedance probability. Indeed, the main theorem of Section 3 illustrates this through a certain class of such processes obtained by using the infinite law of a standard Brownian motion shifted according to the Lebesgue measure as the control measure. For illustrative purposes, a simple kernel with compact support is chosen; and it is shown by evaluating the exact limit that even for this simple kernel

$$
\psi(u)=O\left(u^{(\alpha+1) / 2 p-\alpha}\right) \quad \text { as } u \rightarrow \infty .
$$

Observe that it follows from the particular choice of $\alpha$ that

$$
\frac{\alpha+1}{2}>1,
$$

and, hence, it is clear that the order of magnitude of the exceedance probability is larger in the conservative case.

This observation is indeed consistent with the scheme suggested in [28], and further developed in [3], [4], [24], and [29] to distinguish between short- and long-range dependence of stationary stable processes. All these papers observed a significant difference in the behavior of a functional acting on the sample paths of stationary stable processes when one considers the processes generated by dissipative flows versus the processes generated by conservative flows. It was then suggested that this difference is related to the dependence structure of the underlying stable process, and the processes generated by dissipative flows should be considered short-range dependent, whereas the processes generated by conservative flows are long-range dependent.

Furthermore, our results are consistent with those of [3] and [4], in the sense that if we let $p \downarrow 1$ in the result given in Theorem 1, we obtain the result given in [3, Theorem 3.3.b]; and if we let $p \downarrow 1$ in the result given in Theorem 2, we obtain the result given in [4, Theorem 3.1].

In this paper we analyze the asymptotic behavior of the probability of an integrated stable process exceeding a deterministic power barrier described by $b(t)=\mu t^{p}$ with $\mu>0$ and $p>1$. We can consider a slight generalization by choosing $b(t)$ to be regularly varying with index $p$, i.e. $b(t)=t^{p} L(t)$, where $L(\cdot)$ is a slowly varying function. However, unlike power functions, regularly varying functions are not necessarily monotone and it can be seen in the next section that monotonicity of $b(\cdot)$ is essential in our proofs. To overcome this particular problem in the regularly varying case, we need to introduce generalized inverses and strictly monotone approximations to the barrier function. This in turn prevents us from getting precise asymptotic results with exact constants as in the two main theorems above. Since the principal objective of this paper is to get a qualitative comparison of dissipative and conservative cases to obtain a deeper insight into the probabilistic structure of stable processes and to get exact asymptotic results, we prefer to work with power barriers. 


\section{Proofs}

In this section we present the proofs of the results given earlier in the paper.

\subsection{Proof of Proposition 1}

Provided that (10) holds, Lemma 3.2 of [3] implies that there exists an $\tilde{\varepsilon} \in(0,1)$ such that the process $\tilde{\boldsymbol{Y}}=\{\tilde{Y}(t), t \geq 0\}$ defined by

$$
\tilde{Y}(t):=(t+1)^{\tilde{\varepsilon}-1} S(t), \quad t \geq 0,
$$

is a.s. bounded. So pick $\tilde{\varepsilon} \in(0,1)$ such that $\tilde{\boldsymbol{Y}}$ is a.s. bounded. Next, define a process $\boldsymbol{Y}=\{Y(t), t \geq 0\}$ by

$$
Y(t)=\frac{[\log (b(t)+2)]^{1+\varepsilon}}{b(t)+2} S(t), \quad t \geq 0 .
$$

Note, for any $\varepsilon>0$ and $p>1$, it can be easily verified that $\left[\log \left(t^{p} \mu+2\right)\right]^{1+\varepsilon} /\left(t^{p} \mu+2\right)$ is $o\left((t+1)^{\tilde{\varepsilon}-1}\right)$ as $t \rightarrow \infty$. Then, since $\tilde{\varepsilon}>0$ is picked such that $\tilde{\boldsymbol{Y}}$ is a.s. bounded, we see that, for any $\varepsilon>0, Y$ is a.s. bounded. The result follows from Theorem 4.1 and Remark 4.2 of [8].

\subsection{Proof of Theorem 1}

We first prove a lemma, which lets us work with $\psi_{0}$ given in (9) instead of $\psi$.

Lemma 1. Under the assumptions of Theorem 1, $\psi(u) \sim \psi_{0}(u)$ as $u \rightarrow \infty$.

Proof. Start by observing that $g$ is nonnegative, monotone increasing on $\left(-\infty, K_{l}\right]$, monotone decreasing on $\left[K_{r}, \infty\right)$, and is the smallest of all functions dominating $f$, which satisfy these properties. Thus, it is easy to see that $g \in L^{1}(\mathbb{R}, \mathfrak{B}, \lambda)$ implies $f \in L^{1}(\mathbb{R}, \mathfrak{B}, \lambda)$. Then one can choose a $K \in \mathbb{R}_{+}$such that $\int_{-\infty}^{-K} f(x) \mathrm{d} x$ and $\int_{K}^{\infty} f(x) \mathrm{d} x$ are both smaller than 1 , and, hence,

$$
\begin{aligned}
\left\|h_{t}(\cdot)\right\|_{L^{\alpha}(\mathbb{R}, \mathfrak{B}, \lambda)}^{\alpha} & =\int_{\mathbb{R} \backslash(-K, K+t)} h_{t}^{\alpha}(x) \mathrm{d} x+\int_{(-K, K+t)} h_{t}^{\alpha}(x) \mathrm{d} x \\
& \leq \int_{\mathbb{R}} \int_{0}^{t} f(x-s) \mathrm{d} s \mathrm{~d} x+\int_{(-K, K+t)}\|f(\cdot)\|_{L^{1}(\mathbb{R}, \mathfrak{B}, \lambda)}^{\alpha} \mathrm{d} x \\
& \leq t\|f(\cdot)\|_{L^{1}(\mathbb{R}, \mathfrak{B}, \lambda)}+(2 K+t)\|f(\cdot)\|_{L^{1}(\mathbb{R}, \mathfrak{B}, \lambda)}^{\alpha} .
\end{aligned}
$$

Therefore, $f \in L^{1}(\mathbb{R}, \mathfrak{B}, \lambda)$ implies that $\left\|h_{t}(\cdot)\right\|_{L^{\alpha}(\mathbb{R}, \mathfrak{B}, \lambda)}=O\left(t^{1 / \alpha}\right)$ as $t \rightarrow \infty$. The result follows from Proposition 1 .

A similar argument to that given in the proof of Lemma 1 leads to the following useful observation.

Lemma 2. Under the assumptions of Theorem $1, g \in L^{\alpha}(\mathbb{R}, \mathfrak{B}, \lambda)$.

Proof. Since $g \in L^{1}(\mathbb{R}, \mathfrak{B}, \lambda)$ is nonnegative, monotone increasing on $\left(-\infty, K_{l}\right]$, and monotone decreasing on $\left[K_{r}, \infty\right)$, there exists a finite constant $C>K_{r}-K_{l}$ such that $g(x)<1$ for any $x \in \mathbb{R} \backslash(-C, C)$. Also, without loss of generality, assume that $g\left(K_{l}\right), g\left(K_{r}\right)<\infty$. 
Then

$$
\begin{aligned}
\|g\|_{L^{\alpha}(\mathbb{R}, \mathfrak{B}, \lambda)}^{\alpha} & \leq\|g\|_{L^{1}(\mathbb{R}, \mathfrak{B}, \lambda)}+\|f\|_{L^{\alpha}(\mathbb{R}, \mathfrak{B}, \lambda)}^{\alpha}+\int_{-C}^{K_{l}} g^{\alpha}(x) \mathrm{d} x+\int_{K_{r}}^{C} g^{\alpha}(x) \mathrm{d} x \\
& \leq\|g\|_{L^{1}(\mathbb{R}, \mathfrak{B}, \lambda)}+\|f\|_{L^{\alpha}(\mathbb{R}, \mathfrak{B}, \lambda)}^{\alpha}+2 C\left[g\left(K_{l}\right)+g\left(K_{r}\right)\right] \\
& <\infty
\end{aligned}
$$

yielding the desired result.

Now define $\psi^{*}(u):=\int_{\mathbb{R}} \sup _{t \geq 0}\left[h_{t}(x) /\left(u+\mu t^{p}\right)\right]^{\alpha} \mathrm{d} x, u>0$. Observe that, since $f \geq 0$, (9) reduces to

$$
\frac{2 \psi_{0}(\cdot)}{C_{\alpha}}=\psi^{*}(\cdot)
$$

In the light of Lemma 1 , we focus on $\psi^{*}$ in the rest of the proof of Theorem 1 . We start by showing that

$$
\limsup _{u \rightarrow \infty} u^{\alpha-1 / p} \psi^{*}(u) \leq \frac{\|f\|_{L^{1}(\mathbb{R}, \mathfrak{B}, \lambda)}^{\alpha}}{p \mu^{1 / p}} \mathscr{B}\left(\frac{1}{p}, \alpha-\frac{1}{p}\right) .
$$

To do so, we write

$$
\begin{aligned}
\psi^{*}(u) & =\int_{-\infty}^{K_{l}} \sup _{t \geq 0}\left[\frac{h_{t}(x)}{u+\mu t^{p}}\right]^{\alpha} \mathrm{d} x+\int_{K_{l}}^{K_{r}} \sup _{t \geq 0}\left[\frac{h_{t}(x)}{u+\mu t^{p}}\right]^{\alpha} \mathrm{d} x+\int_{K_{r}}^{\infty} \sup _{t \geq 0}\left[\frac{h_{t}(x)}{u+\mu t^{p}}\right]^{\alpha} \mathrm{d} x \\
& =: \psi_{1}^{*}(u)+\psi_{2}^{*}(u)+\psi_{3}^{*}(u) .
\end{aligned}
$$

We focus on $\psi_{1}^{*}$ first. Note that

$$
\begin{aligned}
\psi_{1}^{*}(u) & \leq \int_{-\infty}^{K_{l}} \sup _{0 \leq t \leq-x}\left[\frac{h_{t}(x)}{u+\mu t^{p}}\right]^{\alpha} \mathrm{d} x+\int_{-\infty}^{K_{l}} \sup _{t \geq-x}\left[\frac{h_{t}(x)}{u+\mu t^{p}}\right]^{\alpha} \mathrm{d} x \\
& =: \psi_{11}^{*}(u)+\psi_{12}^{*}(u) .
\end{aligned}
$$

Since $g \in L^{1}(\mathbb{R}, \mathfrak{B}, \lambda)$ and is monotone increasing on $\left(-\infty, K_{l}\right]$, it is easy to see that $g(x)$ is $o(-1 / x)$ as $x \rightarrow-\infty$. Consequently, for every $\epsilon>0$, there exists $x_{\epsilon} \leq K_{l}$ such that, for any $x<x_{\epsilon}, g(x)<-\epsilon / x$. Fix $\epsilon>0$ and $x_{\epsilon}$. Then pick $u>0$ sufficiently large such that

$$
x_{\epsilon}>-\left(\frac{u}{(p-1) \mu}\right)^{1 / p}=: x_{u} \text {. }
$$

Next note that it follows from the monotonicity of $g$ on $\left(-\infty, K_{l}\right]$ that

$$
\begin{aligned}
\psi_{11}^{*}(u) \leq & \int_{-\infty}^{K_{l}} \sup _{0 \leq t \leq-x}\left[\frac{\int_{0}^{t} g(x-s) \mathrm{d} s}{u+\mu t^{p}}\right]^{\alpha} \mathrm{d} x \\
\leq & \int_{-\infty}^{K_{l}} \sup _{0 \leq t \leq-x}\left[\frac{\operatorname{tg}(x)}{u+\mu t^{p}}\right]^{\alpha} \mathrm{d} x \\
\leq & \int_{-\infty}^{x_{u}} \sup _{0 \leq t \leq-x}\left[\frac{\operatorname{tg}(x)}{u+\mu t^{p}}\right]^{\alpha} \mathrm{d} x+\int_{x_{u}}^{x_{\epsilon}} \sup _{0 \leq t \leq-x}\left[\frac{\operatorname{tg}(x)}{u+\mu t^{p}}\right]^{\alpha} \mathrm{d} x \\
& +\int_{x_{\epsilon}}^{K_{l}} \sup _{0 \leq t \leq-x}\left[\frac{\operatorname{tg}(x)}{u+\mu t^{p}}\right]^{\alpha} \mathrm{d} x \\
= & : \psi_{111}^{*}(u)+\psi_{112}^{*}(u)+\psi_{113}^{*}(u) .
\end{aligned}
$$


Lemma 3. For $x \in\left(-\infty, x_{u}\right]$,

$$
\sup _{0 \leq t \leq-x}\left[\frac{\operatorname{tg}(x)}{u+\mu t^{p}}\right] \leq\left(\frac{p-1}{u}\right)^{(p-1) / p} \mu^{-1 / p} p^{-1} g(x) .
$$

Proof. Define $F(t):=\operatorname{tg}(x) /\left(u+\mu t^{p}\right)$. Observe that, on $t \geq 0, F$ is nonnegative and continuous. Also, note that

$$
\lim _{t \downarrow 0} F(t)=\lim _{t \uparrow \infty} F(t)=0 .
$$

Furthermore, on $t \geq 0$, it is an easy task to verify that the only root of the equation $\mathrm{d} F(t) / \mathrm{d} t=0$ is $t_{0}=x_{u}$. Therefore,

$$
\sup _{0 \leq t \leq-x}\left[\frac{\operatorname{tg}(x)}{u+\mu t^{p}}\right] \leq \sup _{t \geq 0} F(t)=F\left(x_{u}\right),
$$

which completes the proof of the lemma.

Now it follows from the above lemma that

$$
\begin{aligned}
\limsup _{u \rightarrow \infty} u^{\alpha-1 / p} \psi_{111}^{*}(u) & =\limsup _{u \rightarrow \infty} u^{\alpha-1 / p} \int_{-\infty}^{x_{u}} \sup _{0 \leq t \leq-x}\left[\frac{\operatorname{tg}(x)}{u+\mu t^{p}}\right]^{\alpha} \mathrm{d} x \\
& \leq\left[\frac{(p-1)^{(p-1) / p}}{\mu^{1 / p} p}\right]^{\alpha} \limsup _{u \rightarrow \infty} u^{(\alpha-1) / p} \int_{-\infty}^{x_{u}} g^{\alpha}(x) \mathrm{d} x \\
& \leq\left[\frac{\epsilon(p-1)^{(p-1) / p}}{\mu^{1 / p} p}\right]^{\alpha} \limsup _{u \rightarrow \infty} u^{(\alpha-1) / p} \int_{-\infty}^{x_{u}}(-x)^{-\alpha} \mathrm{d} x \\
& =\frac{\epsilon^{\alpha}(p-1)^{\alpha-1 / p}}{(\alpha-1) \mu^{1 / p} p^{\alpha}} .
\end{aligned}
$$

Moreover,

$$
\begin{aligned}
\limsup _{u \rightarrow \infty} u^{\alpha-1 / p} \psi_{112}^{*}(u) & =\limsup _{u \rightarrow \infty} u^{\alpha-1 / p} \int_{x_{u}}^{x_{\epsilon}} \sup _{0 \leq t \leq-x}\left[\frac{\operatorname{tg}(x)}{u+\mu t^{p}}\right]^{\alpha} \mathrm{d} x \\
& \leq \limsup _{u \rightarrow \infty} u^{\alpha-1 / p} \int_{x_{u}}^{x_{\epsilon}}\left[\frac{-x g(x)}{u}\right]^{\alpha} \mathrm{d} x \\
& \leq \epsilon^{\alpha} \limsup _{u \rightarrow \infty} u^{-1 / p}\left(x_{u}-x_{\epsilon}\right) \\
& =\epsilon^{\alpha}[(p-1) \mu]^{-1 / p}
\end{aligned}
$$

Lastly, it follows from Lemma 2 that

$$
\begin{aligned}
\limsup _{u \rightarrow \infty} u^{\alpha-1 / p} \psi_{113}^{*}(u) & \leq \limsup _{u \rightarrow \infty} u^{\alpha-1 / p} \int_{x_{\epsilon}}^{K_{l}}\left[\frac{-x g(x)}{u}\right]^{\alpha} \mathrm{d} x \\
& \leq \limsup _{u \rightarrow \infty} u^{-1 / p}\left(x_{\epsilon}\right)^{\alpha}\|g\|_{L^{\alpha}(\mathbb{R}, \mathfrak{B}, \lambda)}^{\alpha} \\
& =0 .
\end{aligned}
$$


Furthermore, observe that it follows from $f \in L^{1}(\mathbb{R}, \mathfrak{B}, \lambda)$ that, for every $\theta>0$, there exists $x_{\theta} \leq K_{l}$ such that $\int_{-\infty}^{x_{\theta}} f(x) \mathrm{d} x<\theta$. Then

$$
\begin{aligned}
\limsup _{u \rightarrow \infty} u^{\alpha-1 / p} \psi_{12}^{*}(u)= & \limsup _{u \rightarrow \infty} u^{\alpha-1 / p} \int_{-\infty}^{K_{l}} \sup _{t \geq-x}\left[\frac{h_{t}(x)}{u+\mu t^{p}}\right]^{\alpha} \mathrm{d} x \\
\leq & \limsup _{u \rightarrow \infty} u^{\alpha-1 / p} \int_{-\infty}^{x_{\theta}}\left[\frac{\int_{0}^{\infty} f(x-s) \mathrm{d} s}{u+(-x)^{p} \mu}\right]^{\alpha} \mathrm{d} x \\
& +\limsup _{u \rightarrow \infty} u^{\alpha-1 / p} \int_{x_{\theta}}^{K_{l}}\left[\frac{\int_{0}^{\infty} f(x-s) \mathrm{d} s}{u}\right]^{\alpha} \mathrm{d} x \\
\leq & \limsup _{u \rightarrow \infty} u^{\alpha-1 / p} \int_{-\infty}^{x_{\theta}}\left[\frac{\int_{-\infty}^{x} f(y) \mathrm{d} y}{u+(-x)^{p} \mu}\right]^{\alpha} \mathrm{d} x \\
& +\limsup _{u \rightarrow \infty} u^{-1 / p}\left(K_{l}-x_{\theta}\right)\|f\|_{L^{1}(\mathbb{R}, \mathfrak{B}, \lambda)}^{\alpha} \\
\leq & \theta^{\alpha} \limsup _{u \rightarrow \infty} u^{\alpha-1 / p} \int_{-\infty}^{x_{\theta}}\left[u+(-x)^{p} \mu\right]^{-\alpha} \mathrm{d} x .
\end{aligned}
$$

Substituting $y=(-x)^{p} \mu / u$ we have

$$
\begin{aligned}
\limsup _{u \rightarrow \infty} u^{\alpha-1 / p} \psi_{12}^{*}(u) & \leq \frac{\theta^{\alpha}}{p \mu^{1 / p}} \limsup _{u \rightarrow \infty} \int_{\left(-x_{\theta}\right)^{p} \mu / u}^{\infty}(1+y)^{-\alpha} y^{1 / p-1} \mathrm{~d} y \\
& =\frac{\theta^{\alpha}}{p \mu^{1 / p}} \mathcal{B}\left(\frac{1}{p}, \alpha-\frac{1}{p}\right) .
\end{aligned}
$$

Combining (15)-(21) we see that

$$
\limsup _{u \rightarrow \infty} u^{\alpha-1 / p} \psi_{1}^{*}(u) \leq \frac{\epsilon^{\alpha}(p-1)^{\alpha-1 / p}}{(\alpha-1) \mu^{1 / p} p^{\alpha}}+\frac{\epsilon^{\alpha}}{[(p-1) \mu]^{1 / p}}+\frac{\theta^{\alpha} \mathcal{B}(1 / p, \alpha-1 / p)}{p \mu^{1 / p}} .
$$

Letting $\epsilon \downarrow 0$ and $\theta \downarrow 0$ we conclude that

$$
\psi_{1}^{*}(u) \sim o\left(u^{1 / p-\alpha}\right) \quad \text { as } u \rightarrow \infty .
$$

Next note that

$$
\psi_{2}^{*}(u)=\int_{K_{l}}^{K_{r}} \sup _{t \geq 0}\left[\frac{h_{t}(x)}{u+\mu t^{p}}\right]^{\alpha} \mathrm{d} x \leq u^{-\alpha}\left(K_{r}-K_{l}\right)\|f\|_{L^{1}(\mathbb{R}, \mathfrak{B}, \lambda)}^{\alpha},
$$

and, hence,

$$
\psi_{2}^{*}(u) \sim o\left(u^{1 / p-\alpha}\right) \quad \text { as } u \rightarrow \infty .
$$

Before proceeding with $\psi_{3}^{*}$ we recall that there exists a monotone function $g_{\mathrm{rv}}$, which is regularly varying at infinity with index $-q<-1$, which dominates $g$ for all $x \geq K_{r}$. By a characterization theorem due to Karamata (see, for instance, [7, Theorem 1.4.1]) we can write $g_{\mathrm{rv}}(x)=x^{-q} l(x)$, where $l(\cdot)$ is a slowly varying function at infinity. Moreover, it follows from [7, Proposition 1.5.10] that

$$
I_{\mathrm{rv}}:=\int_{K_{r}}^{\infty} g_{\mathrm{rv}}(x) \mathrm{d} x<\infty .
$$


In particular, for any $\vartheta \in(0,1)$ there exists $y_{\vartheta} \geq K_{r}$ such that

$$
\int_{x}^{\infty} g_{\text {rv }}(s) \mathrm{d} s<\vartheta \quad \text { for any } x \geq y_{\vartheta} .
$$

Fix $\vartheta \in(0,1)$ and $y_{\vartheta} \geq K_{r}$, and write

$$
\begin{aligned}
\psi_{3}^{*}(u) \leq & \int_{K_{r}}^{y_{\vartheta}} \sup _{t \geq 0}\left[\frac{h_{t}(x)}{u+\mu t^{p}}\right]^{\alpha} \mathrm{d} x+\int_{y_{\vartheta}}^{\infty} \sup _{t \geq x-y_{\vartheta}}\left[\frac{h_{t}(x)}{u+\mu t^{p}}\right]^{\alpha} \mathrm{d} x \\
& +\int_{y_{\vartheta}}^{\infty} \sup _{0 \leq t \leq x-y_{\vartheta}}\left[\frac{\int_{x-t}^{x} g_{r v}(s) \mathrm{d} s}{u+\mu t^{p}}\right]^{\alpha} \mathrm{d} x \\
= & : \psi_{31}^{*}(u)+\psi_{32}^{*}(u)+\psi_{33}^{*}(u) .
\end{aligned}
$$

We immediately see that

$$
\limsup _{u \rightarrow \infty} u^{\alpha-1 / p} \psi_{31}^{*}(u) \leq \limsup _{u \rightarrow \infty} u^{-1 / p}\left(y_{\vartheta}-K_{r}\right)\|f\|_{L^{1}(\mathbb{R}, \mathfrak{B}, \lambda)}^{\alpha}=0 .
$$

Also, it is easy to see that

$$
\begin{aligned}
\psi_{32}^{*}(u) & \leq\|f\|_{L^{1}(\mathbb{R}, \mathfrak{B}, \lambda)}^{\alpha} \int_{y_{\vartheta}}^{\infty}\left[u+\left(x-y_{\vartheta}\right)^{p} \mu\right]^{-\alpha} \mathrm{d} x \\
& =\frac{\|f\|_{L^{1}(\mathbb{R}, \mathfrak{B}, \lambda)}^{\alpha}}{p \mu^{1 / p}} \mathscr{B}\left(\frac{1}{p}, \alpha-\frac{1}{p}\right) u^{1 / p-\alpha}
\end{aligned}
$$

and, hence,

$$
\limsup _{u \rightarrow \infty} u^{\alpha-1 / p} \psi_{32}^{*}(u) \leq \frac{\|f\|_{L^{1}(\mathbb{R}, \mathfrak{B}, \lambda)}^{\alpha}}{p \mu^{1 / p}} \mathscr{B}\left(\frac{1}{p}, \alpha-\frac{1}{p}\right) .
$$

Next we will need the following lemma.

Lemma 4. For $\varepsilon \in(0,(p-1) / p)$ and $u>0$, let

$$
y_{u(-)}:=\left(\frac{u}{[(1+\varepsilon) p-1] \mu}\right)^{1 / p} \text { and } y_{u(+)}:=\left(\frac{u}{[(1-\varepsilon) p-1] \mu}\right)^{1 / p} .
$$

Then, for any $\varepsilon \in(0,(p-1) / p)$, there exists $y_{\varepsilon}>y_{\vartheta}+y_{u(+)}$ such that, for any $x>y_{\varepsilon}$,

$$
\sup _{t \in\left[0, x-y_{\vartheta}\right]} \frac{\int_{x-t}^{x} g_{\mathrm{rv}}(s) \mathrm{d} s}{u+t^{p} \mu}=\sup _{t \in\left[y_{u(-)}, y_{u(+)}\right]} \frac{\int_{x-t}^{x} g_{\mathrm{rv}}(s) \mathrm{d} s}{u+t^{p} \mu} .
$$

Proof. Start by defining

$$
G_{\mathrm{rv}}(x, t):=\frac{\int_{x-t}^{x} g_{\mathrm{rv}}(s) \mathrm{d} s}{u+t^{p} \mu}, \quad x \geq y_{\vartheta}, t \in\left[0, x-y_{\vartheta}\right] .
$$

Then, for $t \in\left(0, x-y_{\vartheta}\right)$,

$$
\frac{\partial G_{\mathrm{rv}}(x, t)}{\partial t}=\frac{g_{r v}(x-t)\left[\left(u+t^{p} \mu\right)-p \mu t^{p-1} \int_{x-t}^{x} g_{\mathrm{rv}}(s) \mathrm{d} s / g_{\mathrm{rv}}(x-t)\right]}{\left(u+t^{p} \mu\right)^{2}} .
$$


Observe that it is a direct consequence of Karamata's theorem (see, for instance, [7, Proposition 1.5.10]) that, as $x \rightarrow \infty$,

$$
\frac{\int_{x-t}^{x} g_{\mathrm{rv}}(s) \mathrm{d} s}{g_{\mathrm{rv}}(x-t)} \sim \frac{1}{q-1}\left(\frac{(x-t)^{-q+1}-x^{-q+1}}{(x-t)^{-q}}\right) .
$$

But then, by l'Hôpital's rule,

$$
\frac{\int_{x-t}^{x} g_{\mathrm{rv}}(s) \mathrm{d} s}{g_{\mathrm{rv}}(x-t)} \sim \frac{1-((x-t) / x)^{q-1}}{(q-1)(x-t)^{-1}} \sim \frac{t(1-t / x)^{q-2} x^{-2}}{(x-t)^{-2}} \sim t\left(1-\frac{t}{x}\right)^{q} \sim t .
$$

In particular, for any $\varepsilon \in(0,(p-1) / p)$, there exists $y_{\varepsilon}$ (which, without loss of generality, could be picked to be greater than $\left.y_{\vartheta}+y_{u(+)}\right)$ such that, for any $x>y_{\varepsilon}$,

$$
(1-\varepsilon) t \leq \frac{\int_{x-t}^{x} g_{\mathrm{rv}}(s) \mathrm{d} s}{g_{\mathrm{rv}}(x-t)} \leq(1+\varepsilon) t,
$$

and, hence,

$$
\frac{\partial G_{\mathrm{rv}}(x-t)}{\partial t} \geq \frac{g_{r v}(x-t)\left\{u-t^{p} \mu[(1+\varepsilon) p-1]\right\}}{\left(u+t^{p} \mu\right)^{2}}
$$

and

$$
\frac{\partial G_{\mathrm{rv}}(x-t)}{\partial t} \leq \frac{g_{\mathrm{rv}}(x-t)\left\{u-t^{p} \mu[(1-\varepsilon) p-1]\right\}}{\left(u+t^{p} \mu\right)^{2}} .
$$

Consequently, we see that, for $t \leq y_{u(-)}, \partial G_{\mathrm{rv}}(x-t) / \partial t \geq 0$, and, for $t \geq y_{u(+)}$, $\partial G_{\mathrm{rv}}(x-t) / \partial t \leq 0$, which gives the desired result.

Now fix $\varepsilon \in(0,(p-1) / p)$. It follows from Lemma 4 and the monotonicity of $g_{\mathrm{rv}}$ that, for any $u>0$,

$$
\begin{aligned}
\psi_{33}^{*}(u) & =\int_{y_{\vartheta}}^{\infty} \sup _{0 \leq t \leq x-y_{\vartheta}}\left[\frac{\int_{x-t}^{x} g_{\mathrm{rv}}(s) \mathrm{d} s}{u+\mu t^{p}}\right]^{\alpha} \mathrm{d} x \\
& \leq u^{-\alpha} \vartheta^{\alpha}\left(y_{\varepsilon}-y_{\vartheta}\right)+\int_{y_{\varepsilon}}^{\infty} \sup _{t \in\left[y_{u(-)}, y_{u(+)}\right]}\left[\frac{\int_{x-t}^{x} g_{\mathrm{rv}}(s) \mathrm{d} s}{u+\mu t^{p}}\right]^{\alpha} \mathrm{d} x \\
& \leq u^{-\alpha} \vartheta^{\alpha}\left(y_{\varepsilon}-y_{\vartheta}\right)+\int_{y_{\varepsilon}}^{\infty}\left[\frac{\int_{x-y_{u(+)}}^{x} g_{\mathrm{rv}}(s) \mathrm{d} s}{u+\mu\left(y_{u(-)}\right)^{p}}\right]^{\alpha} \mathrm{d} x \\
& \leq u^{-\alpha} \vartheta^{\alpha}\left(y_{\varepsilon}-y_{\vartheta}\right)+\frac{y_{u(+)}}{u^{\alpha}}\left[\frac{(1+\varepsilon) p-1}{(1+\varepsilon) p}\right]^{\alpha} \int_{y_{\varepsilon}-y_{u(+)}}^{\infty} g_{\mathrm{rv}}(x) \mathrm{d} x \\
& \leq u^{-\alpha} \vartheta^{\alpha}\left(y_{\varepsilon}-y_{\vartheta}\right)+u^{-\alpha} y_{u(+)} \vartheta\left[\frac{(1+\varepsilon) p-1}{(1+\varepsilon) p}\right]^{\alpha} .
\end{aligned}
$$

Therefore,

$$
\limsup _{u \rightarrow \infty} u^{\alpha-1 / p} \psi_{33}^{*}(u) \leq \vartheta\left[\frac{(1+\varepsilon) p-1}{[(1-\varepsilon) p-1]^{1 /(\alpha p)}(1+\varepsilon) p}\right]^{\alpha} .
$$

Combining (24), (25), (26), and (27), and letting $\vartheta \downarrow 0$, we conclude that

$$
\limsup _{u \rightarrow \infty} u^{\alpha-1 / p} \psi_{3}^{*}(u) \leq \frac{\|f\|_{L^{1}(\mathbb{R}, \mathfrak{B}, \lambda)}^{\alpha}}{p \mu^{1 / p}} \mathscr{B}\left(\frac{1}{p}, \alpha-\frac{1}{p}\right) .
$$


Finally, combining (14), (22), (23), and (28), we have

$$
\limsup _{u \rightarrow \infty} u^{\alpha-1 / p} \psi^{*}(u) \leq \frac{\|f\|_{L^{1}(\mathbb{R}, \mathfrak{B}, \lambda)}^{\alpha}}{p \mu^{1 / p}} \mathscr{B}\left(\frac{1}{p}, \alpha-\frac{1}{p}\right) .
$$

Next we will show that

$$
\liminf _{u \rightarrow \infty} u^{\alpha-1 / p} \psi^{*}(u) \geq \frac{\|f\|_{L^{1}(\mathbb{R}, \mathfrak{B}, \lambda)}^{\alpha}}{p \mu^{1 / p}} \mathscr{B}\left(\frac{1}{p}, \alpha-\frac{1}{p}\right) .
$$

Recall that, under the assumptions of the theorem, $f \in L^{1}(\mathbb{R}, \mathfrak{B}, \lambda)$. Since $f$ is nonnegative, for any $y \in \mathbb{R}$ and any $\delta \in(0,1)$, there exists $y_{\delta}>y$ such that

$$
\int_{y}^{y_{\delta}} f(x) \mathrm{d} x \geq(1-\epsilon) \int_{y}^{\infty} f(x) \mathrm{d} x .
$$

Then, for any such $y$ and $y_{\delta}$, we have

$$
\begin{aligned}
\psi^{*}(u) & \geq \int_{y_{\delta}}^{\infty} \sup _{t \geq 0}\left[\frac{\int_{x-t}^{x} f(s) \mathrm{d} s}{u+t^{p} \mu}\right]^{\alpha} \mathrm{d} x \\
& \geq \int_{y_{\delta}}^{\infty}\left[\frac{\int_{y}^{x} f(s) \mathrm{d} s}{u+(x-y)^{p} \mu}\right]^{\alpha} \mathrm{d} x \\
& \geq\left[\int_{y}^{y_{\delta}} f(s) \mathrm{d} s\right]^{\alpha} \int_{y_{\delta}}^{\infty}\left[u+(x-y)^{p} \mu\right]^{-\alpha} \mathrm{d} x \\
& \geq \frac{u^{1 / p-\alpha}\left[(1-\delta) \int_{y}^{\infty} f(s) \mathrm{d} s\right]^{\alpha}}{p \mu^{1 / p}} \int_{\left(y_{\delta}-y\right)^{p} \mu / u}^{\infty}(1+x)^{-\alpha} x^{1 / p-1} \mathrm{~d} x,
\end{aligned}
$$

and, hence,

$$
\liminf _{u \rightarrow \infty} u^{\alpha-1 / p} \psi^{*}(u) \geq \frac{\left[(1-\delta) \int_{y}^{\infty} f(s) \mathrm{d} s\right]^{\alpha}}{p \mu^{1 / p}} \mathscr{B}\left(\frac{1}{p}, \alpha-\frac{1}{p}\right) .
$$

Letting $\delta \downarrow 0$ and $y \downarrow-\infty$ and combining with (29),

$$
\lim _{u \rightarrow \infty} u^{\alpha-1 / p} \psi^{*}(u)=\frac{\|f\|_{L^{1}(\mathbb{R}, \mathfrak{B}, \lambda)}^{\alpha}}{p \mu^{1 / p}} \mathcal{B}\left(\frac{1}{p}, \alpha-\frac{1}{p}\right) .
$$

Then (13) and Lemma 1 conclude the proof of the theorem.

\subsection{Proof of Theorem 2}

Start by defining

$$
\frac{2 \psi_{0}(u)}{C_{\alpha}}:=\int_{C(\mathbb{R})} \sup _{t \geq 0}\left[\frac{h_{t}(\boldsymbol{x})}{u+t^{p} \mu}\right]^{\alpha} m(\mathrm{~d} \boldsymbol{x})=\int_{\mathbb{R}} \mathrm{E}\left[\sup _{t \geq 0}\left[\frac{\int_{0}^{t} \varphi(B(s)+y) \mathrm{d} s}{u+t^{p} \mu}\right]^{\alpha}\right] \mathrm{d} y,
$$

where $\mu>0, p>1$, and $C_{\alpha}=\left(\int_{0}^{\infty} x^{-\alpha} \sin x \mathrm{~d} x\right)^{-1}$, and observing the following lemma.

Lemma 5. We have $\psi(u) \sim \psi_{0}(u)$ as $u \rightarrow \infty$. 
Proof. It follows from Lemma 3.2 of [4] that there exists $\tilde{\varepsilon} \in(0,1)$ such that the process $\tilde{\boldsymbol{Y}}=\left\{\tilde{Y}(t):=(t+1)^{\tilde{\varepsilon}-1} S(t), t \geq 0\right\}$ is a.s. bounded. Then, following the same steps as in the proof of Proposition 1 gives the desired result.

Now let $\psi^{*}$ be as in (13). In the light of the above lemma we focus on $\psi^{*}$ instead of $\psi$. So, for $u>0$, write

$$
\begin{aligned}
\psi^{*}(u)= & \int_{\mathbb{R}} \mathrm{E}\left[\sup _{t>0}\left[\frac{\int_{0}^{t} \varphi(B(s)+y) \mathrm{d} s}{u+t^{p} \mu}\right]^{\alpha}\right] \mathrm{d} y \\
= & \int_{-1}^{1} \mathrm{E}\left[\sup _{t>0}\left[\frac{\int_{0}^{t} \varphi(B(s)+y) \mathrm{d} s}{u+t^{p} \mu}\right]^{\alpha}\right] \mathrm{d} y \\
& +\int_{-\infty}^{-1} \mathrm{E}\left[\sup _{t>0}\left[\frac{\int_{0}^{t} \varphi(B(s)+y) \mathrm{d} s}{u+t^{p} \mu}\right]^{\alpha}\right] \mathrm{d} y \\
& +\int_{1}^{\infty} \mathrm{E}\left[\sup _{t>0}\left[\frac{\int_{0}^{t} \varphi(B(s)+y) \mathrm{d} s}{u+t^{p} \mu}\right]^{\alpha}\right] \mathrm{d} y \\
= & : \psi_{1}^{*}(u)+\psi_{2}^{*}(u)+\psi_{3}^{*}(u) .
\end{aligned}
$$

Then observe, by Hölder's inequality and Fubini's theorem, that

$$
\begin{aligned}
\psi_{1}^{*}(u) & =\int_{-1}^{1} \mathrm{E}\left[\sup _{t>0}\left[\frac{\int_{\mathbb{R}} \varphi(x+y) L(x, t) \mathrm{d} x}{u+t^{p} \mu}\right]^{\alpha}\right] \mathrm{d} y \\
& \leq \int_{-1}^{1} \mathrm{E}\left[\sup _{t>0}\left[\frac{\int_{-1-y}^{1-y} L(x, t) \mathrm{d} x}{u+t^{p} \mu}\right]^{\alpha}\right] \mathrm{d} y \\
& \leq 2 \mathrm{E}\left[\sup _{t>0}\left[\frac{\int_{-2}^{2} L(x, t) \mathrm{d} x}{u+t^{p} \mu}\right]^{\alpha}\right] \\
& \leq 2^{2 \alpha-1} \sup _{t>0}\left[\frac{\sqrt{t}}{u+t^{p} \mu}\right]_{-2}^{\alpha} \int_{-2}^{2}\left[\sup _{t>0} L^{\alpha}\left(\frac{x}{\sqrt{t}}, 1\right)\right] \mathrm{d} x \\
& =2^{\alpha-1}\left\{\frac{[u /(2 p-1) \mu]^{1 / 2 p}}{p u}\right\}_{-2}^{\alpha} \mathrm{E}\left[\sup _{t>0}^{\alpha} L^{\alpha}\left(\frac{x}{\sqrt{t}}, 1\right)\right] \mathrm{d} x .
\end{aligned}
$$

Now recall that all moments of the supremum of the local time $L(x, t)$ over all real $x$ are finite. Consequently, it follows from the above observation that, for some positive constant $c_{0}$,

$$
\limsup _{u \rightarrow \infty} u^{(2 p \alpha-\alpha-p) / 2 p} \psi_{1}^{*}(u) \leq c_{0} \limsup _{u \rightarrow \infty} u^{(2 p \alpha-\alpha-p) / 2 p}\left\{\frac{u^{1 / 2 p-1}}{p[(2 p-1) \mu]^{1 / 2 p}}\right\}^{\alpha}=0 .
$$

We will need the following two lemmas before we can proceed with $\psi_{2}^{*}$ and $\psi_{3}^{*}$.

Lemma 6. For any $y \in \mathbb{R}$, as $u \rightarrow \infty$,

$$
g(u, y):=\mathrm{E}\left[\sup _{t \geq 0}\left[\frac{\int_{0}^{t} \varphi(B(s)+y) \mathrm{d} s}{u+t^{p}}\right]^{\alpha}\right] \sim u^{-\alpha(1-1 / 2 p)} \mathrm{E}\left[\sup _{t \geq 0}\left[\frac{L\left(0, t^{1 / p}\right)}{1+t}\right]^{\alpha}\right] .
$$

Proof. Fix $y \in \mathbb{R}$ and $K>0$, and define

$$
g_{1}(u, y):=\mathrm{E}\left[\sup _{t \geq(u K)^{1 / p}}\left[\frac{\int_{0}^{t} \varphi(B(s)+y) \mathrm{d} s}{u+t^{p}}\right]^{\alpha}\right]
$$


and

$$
g_{2}(u, y):=\mathrm{E}\left[\sup _{0 \leq t \leq(u K)^{1 / p}}\left[\frac{\int_{0}^{t} \varphi(B(s)+y) \mathrm{d} s}{u+t^{p}}\right]^{\alpha}\right] .
$$

It follows from Hölder's inequality and Fubini's theorem that

$$
\begin{aligned}
g_{1}(u, y) & \leq \sum_{j=1}^{\infty} \mathrm{E}\left[\sup _{\left(u K 2^{j-1}\right)^{1 / p} \leq t \leq\left(u K 2^{j}\right)^{1 / p}}\left[\frac{\int_{0}^{t} \varphi(B(s)+y) \mathrm{d} s}{u+t^{p}}\right]^{\alpha}\right] \\
& \leq\left(\frac{u}{2}\right)^{-\alpha} \sum_{j=1}^{\infty} \mathrm{E}\left[\left(\frac{\int_{0}^{\left(u K 2^{j}\right)^{1 / p}} \varphi(B(s)+y) \mathrm{d} s}{K 2^{j}}\right)^{\alpha}\right] \\
& \leq 2^{\alpha}(u K)^{-\alpha(1-1 / 2 p)} \sum_{j=1}^{\infty} 2^{-j \alpha(1-1 / 2 p)} \int_{-1-y}^{1-y} \mathrm{E}\left[L^{\alpha}\left(\frac{x}{\left(u K 2^{j}\right)^{1 / 2 p}}, 1\right)\right] \mathrm{d} x .
\end{aligned}
$$

Since all moments of the supremum of the local time $L(x, t)$ over all real $x$ are finite, the sum on the right-hand side converges, and, hence,

$$
\lim _{K \uparrow \infty} \limsup _{u \rightarrow \infty} u^{\alpha(1-1 / 2 p)} g_{1}(u, y)=0 .
$$

Next observe that, for any continuity point $z \geq 0$ of the distribution of

$$
\sup _{0 \leq s \leq K} \frac{L\left(0, s^{1 / p}\right)}{1+s},
$$

it follows from the argument given in [10, p. 52] that, as $u \rightarrow \infty$,

$$
\begin{aligned}
\mathrm{P}\left(\sup _{0 \leq t \leq(u K)^{1 / p}} \frac{u^{1-1 / 2 p} \int_{0}^{t} \varphi(B(s)+y) \mathrm{d} s}{u+t^{p}} \geq z\right) \\
\quad=\mathrm{P}\left(u^{-1 / 2 p} \int_{0}^{(u s)^{1 / p}} \varphi(B(s)+y) \mathrm{d} s \geq(1+s) z \text { for some } 0 \leq s \leq K\right) \\
\sim \mathrm{P}\left(L\left(0, s^{1 / p}\right) \geq(1+s) z \text { for some } 0 \leq s \leq K\right) \\
\quad=\mathrm{P}\left(\sup _{0 \leq s \leq K} \frac{L\left(0, s^{1 / p}\right)}{1+s} \geq z\right) .
\end{aligned}
$$

Hence, we conclude that, as $u \rightarrow \infty$,

$$
\sup _{0 \leq t \leq(u K)^{1 / p}} \frac{u^{1-1 / 2 p} \int_{0}^{t} \varphi(B(s)+y) \mathrm{d} s}{u+t^{p}} \Rightarrow \sup _{0 \leq t \leq K} \frac{L\left(0, t^{1 / p}\right)}{1+t},
$$

where ' $\Rightarrow$ ' indicates weak convergence. Then, by the continuous mapping theorem,

$$
\left[\sup _{0 \leq t \leq(u K)^{1 / p}} \frac{u^{1-1 / 2 p} \int_{0}^{t} \varphi(B(s)+y) \mathrm{d} s}{u+t^{p}}\right]^{\alpha} \Rightarrow\left[\sup _{0 \leq t \leq K} \frac{L\left(0, t^{1 / p}\right)}{1+t}\right]^{\alpha} .
$$

Also, observe that

$$
\sup _{0 \leq t \leq(u K)^{1 / p}} \frac{u^{1-1 / 2 p} \int_{0}^{t} \varphi(B(s)+y) \mathrm{d} s}{u+t^{p}} \leq u^{-1 / 2 p} \int_{-1-y}^{1-y} L\left(x,(u K)^{1 / p}\right) \mathrm{d} x,
$$


and, hence, for any $\delta>0$, by Hölder's inequality and (12) we have

$$
\begin{aligned}
& {\left[\sup _{0 \leq t \leq(u K)^{1 / p}} \frac{u^{1-1 / 2 p} \int_{0}^{t} \varphi(B(s)+y) \mathrm{d} s}{u+t^{p}}\right]^{\alpha+\delta}} \\
& \quad \leq 2^{\alpha+\delta-1} \int_{-1-y}^{1-y} L^{\alpha+\delta}\left(\frac{x}{u^{1 / 2 p}}, K^{1 / p}\right) \mathrm{d} x .
\end{aligned}
$$

It follows from Fubini's theorem that

$$
\begin{aligned}
& \sup _{u>0} \mathrm{E}\left[\left.\sup _{0 \leq t \leq(u K)^{1 / p}} \frac{u^{1-1 / 2 p} \int_{0}^{t} \varphi(B(s)+y) \mathrm{d} s}{u+t^{p}}\right|^{\alpha+\delta}\right] \\
& \quad \leq 2^{\alpha+\delta-1} \int_{-1-y}^{1-y} \mathrm{E}\left[\sup _{u>0} L^{\alpha+\delta}\left(\frac{x}{u^{1 / 2 p}}, K^{1 / p}\right)\right] \mathrm{d} x \\
& \quad<\infty
\end{aligned}
$$

since all moments of the supremum of the local time $L(x, t)$ over all real $x$ are finite. Thus, it follows from the conditions discussed in [25, p. 184] that the family

$$
\left\{\left[\sup _{0 \leq t \leq(u K)^{1 / p}} \frac{u^{1-1 / 2 p} \int_{0}^{t} \varphi(B(s)+y) \mathrm{d} s}{u+t^{p}}\right]^{\alpha}\right\}_{u>0}
$$

is uniformly integrable. Combining this with (33), [25, Theorem 6.6.1] gives

$$
\lim _{u \rightarrow \infty} u^{\alpha(1-1 / 2 p)} g_{2}(u, y)=\mathrm{E}\left[\left(\sup _{0 \leq t \leq K} \frac{L\left(0, t^{1 / p}\right)}{1+t}\right)^{\alpha}\right],
$$

and, therefore,

$$
\lim _{K \uparrow \infty} \lim _{u \rightarrow \infty} u^{\alpha(1-1 / 2 p)} g_{2}(u, y)=\mathrm{E}\left[\left(\sup _{t \geq 0} \frac{L\left(0, t^{1 / p}\right)}{1+t}\right)^{\alpha}\right] .
$$

Finally, noting that $g_{2}(u, y) \leq g(u, y) \leq g_{1}(u, y)+g_{2}(u, y)$, and recalling (32), gives the desired result.

Lemma 7. For any $y \in \mathbb{R}$, as $u \rightarrow \infty$,

$$
\begin{aligned}
G(u, y) & :=\int_{0}^{\infty} \mathrm{E}\left[\sup _{t \geq 0}\left[\frac{\int_{0}^{t} \varphi(B(s)+y) \mathrm{d} s}{u+(t+v)^{p}}\right]^{\alpha}\right] v^{-1 / 2} \mathrm{~d} v \\
& \sim \frac{u^{-(2 p \alpha-\alpha-1) / 2 p}}{p} \mathrm{E}\left[\left(\sup _{t \geq 0} \frac{L\left(0, t^{1 / p}\right)}{1+t}\right)^{\alpha}\right] \mathscr{B}\left(\frac{1}{2 p}, \frac{2 p \alpha-\alpha-1}{2 p}\right),
\end{aligned}
$$

where $\mathcal{B}(\cdot, \cdot)$ is the beta function.

Proof. Fix $y \in \mathbb{R}$ and $K>0$, and define

$$
G_{1}(u, y):=\int_{0}^{(u K)^{1 / p}} \mathrm{E}\left[\sup _{t \geq 0}\left(\frac{\int_{0}^{t} \varphi(B(s)+y) \mathrm{d} s}{u+(t+v)^{p}}\right)^{\alpha}\right] v^{-1 / 2} \mathrm{~d} v
$$


and

$$
G_{2}(u, y):=\int_{(u K)^{1 / p}}^{\infty} \mathrm{E}\left[\sup _{t \geq 0}\left(\frac{\int_{0}^{t} \varphi(B(s)+y) \mathrm{d} s}{u+(t+v)^{p}}\right)^{\alpha}\right] v^{-1 / 2} \mathrm{~d} v .
$$

Let $g(u, y)$ be as in Lemma 6. Start by noting that

$$
\begin{aligned}
\int_{0}^{(u K)^{1 / p}} & {\left[\inf _{t \geq 0} \frac{u+v^{p}+t^{p}}{u+(t+v)^{p}}\right]^{\alpha} g\left(u+v^{p}, y\right) v^{-1 / 2} \mathrm{~d} v } \\
\leq & G_{1}(u, y) \\
\leq & \int_{0}^{(u K)^{1 / p}}\left[\sup \frac{u+v^{p}+t^{p}}{u+(t+v)^{p}}\right]^{\alpha} g\left(u+v^{p}, y\right) v^{-1 / 2} \mathrm{~d} v .
\end{aligned}
$$

But

$$
\sup _{t \geq 0} \frac{u+v^{p}+t^{p}}{u+(t+v)^{p}} \leq 1 \quad \text { and } \quad \lim _{u \rightarrow \infty} \inf _{t \geq 0} \frac{u+v^{p}+t^{p}}{u+(t+v)^{p}}=1 .
$$

Then it follows from Lemma 6 that, as $u \rightarrow \infty$,

$$
\begin{aligned}
G_{1}(u, y) & \sim \mathrm{E}\left[\sup _{t \geq 0}\left[\frac{L\left(0, t^{1 / p}\right)}{1+t}\right]^{\alpha}\right] \int_{0}^{(u K)^{1 / p}}\left(u+v^{p}\right)^{-\alpha(1-1 / 2 p)} v^{-1 / 2} \mathrm{~d} v \\
& =\frac{u^{-(2 p \alpha-\alpha-1) / 2 p}}{p} \mathrm{E}\left[\sup _{t \geq 0}\left[\frac{L\left(0, t^{1 / p}\right)}{1+t}\right]^{\alpha}\right] \int_{0}^{K}(1+z)^{-\alpha(1-1 / 2 p)} z^{1 / 2 p-1} \mathrm{~d} z .
\end{aligned}
$$

Thus,

$$
\lim _{K \uparrow \infty} \lim _{u \rightarrow \infty} u^{(2 p \alpha-\alpha-1) / 2 p} G_{1}(u, y)=\frac{\mathcal{B}(1 / 2 p,(2 p \alpha-\alpha-1) / 2 p)}{p} \mathrm{E}\left[\sup _{t \geq 0}\left[\frac{L\left(0, t^{1 / p}\right)}{1+t}\right]^{\alpha}\right] .
$$

Furthermore, it follows from Lemma 6 again that, for any $\epsilon>0$, there exists $u_{\epsilon}$ such that, for $u>u_{\epsilon}$,

$$
\begin{aligned}
G_{2}(u, y) & \leq \int_{(u K)^{1 / p}}^{\infty} g\left(v^{p}, y\right) v^{-1 / 2} \mathrm{~d} v \\
& \leq \frac{(1+\epsilon)(u K)^{-(2 p \alpha-\alpha-1) / 2 p}}{p((2 p \alpha-\alpha-1) / 2 p)} \mathrm{E}\left[\sup _{t \geq 0}\left(\frac{L\left(0, t^{1 / p}\right)}{1+t}\right)^{\alpha}\right],
\end{aligned}
$$

and, hence,

$$
\lim _{K \uparrow \infty} \limsup _{u \rightarrow \infty} u^{-(2 p \alpha-\alpha-1) / 2 p} G_{2}(u, y)=0 .
$$

Combining (34) and (35), and observing that $G_{1}(u, y) \leq G(u, y) \leq G_{1}(u, y)+G_{2}(u, y)$, concludes the proof of the lemma.

Lemma 7 will be our key tool in dealing with $\psi_{2}^{*}$ and $\psi_{3}^{*}$. First concentrate on $\psi_{2}^{*}$.

Let $\tau[y]:=\inf \{t \geq 0 ; B(t)=y\}$ be the first passage time of level $y \in \mathbb{R}$. Note that

$$
\begin{aligned}
\psi_{2}^{*}(u) & =\int_{-\infty}^{-1} \mathrm{E}\left[\sup _{t>\tau[-1-y]}\left[\frac{\int_{0}^{t} \varphi(B(s)+y) \mathrm{d} s}{u+t^{p} \mu}\right]^{\alpha}\right] \mathrm{d} y \\
& =\int_{-\infty}^{-1} \mathrm{E}\left[\sup _{t>0}\left[\frac{\int_{\tau[-1-y]}^{t+\tau[-1-y]} \varphi(B(s)+y) \mathrm{d} s}{u+(t+\tau[-1-y])^{p} \mu}\right)^{\alpha}\right] \mathrm{d} y .
\end{aligned}
$$


It is known that, for $v>0$ and $y \in \mathbb{R}$,

$$
\mathrm{P}(\tau[y] \in \mathrm{d} v)=\frac{|y|}{\sqrt{2 \pi v^{3}}} \exp \left(-\frac{y^{2}}{2 v}\right) \mathrm{d} v
$$

(cf. [13, p. 80]). Then, conditioning on $\tau[-1-y]$, we see that the following is a direct consequence of the strong Markov property for Brownian motion and Fubini's theorem:

$$
\begin{aligned}
\psi_{2}^{*}(u) & =\int_{-\infty}^{-1} \int_{0}^{\infty} \mathrm{E}\left[\sup _{t>0}\left[\frac{\int_{0}^{t} \varphi(B(s)-1) \mathrm{d} s}{u+(t+v)^{p} \mu}\right]^{\alpha}\right] \mathrm{P}(\tau[-1-y] \in \mathrm{d} v) \mathrm{d} y \\
& =\int_{0}^{\infty} \mathrm{E}\left[\sup _{t>0}\left[\frac{\int_{0}^{t} \varphi(B(s)-1) \mathrm{d} s}{u+(t+v)^{p} \mu}\right]^{\alpha}\right] \int_{-\infty}^{-1} \frac{-1-y}{\sqrt{2 \pi v^{3}}} \exp \left(-\frac{(-1-y)^{2}}{2 v}\right) \mathrm{d} y \mathrm{~d} v \\
& =\frac{1}{\mu^{\alpha} \sqrt{2 \pi}} \int_{0}^{\infty} \mathrm{E}\left[\sup _{t>0}\left[\frac{\int_{0}^{t} \varphi(B(s)-1) \mathrm{d} s}{u / \mu+(t+v)^{p}}\right]^{\alpha}\right] v^{-1 / 2} \mathrm{~d} v \\
& =\frac{G(u / \mu,-1)}{\mu^{\alpha} \sqrt{2 \pi}} .
\end{aligned}
$$

Similarly, we can easily show that

$$
\begin{aligned}
\psi_{3}^{*}(u) & =\int_{1}^{\infty} \int_{0}^{\infty} \mathrm{E}\left[\sup _{t>0}\left[\frac{\int_{0}^{t} \varphi(B(s)+1) \mathrm{d} s}{u+(t+v)^{p} \mu}\right]^{\alpha}\right] \mathrm{P}(\tau[1-y] \in \mathrm{d} v) \mathrm{d} y \\
& =\frac{G(u / \mu, 1)}{\mu^{\alpha} \sqrt{2 \pi}} .
\end{aligned}
$$

Therefore, recalling (30), (31), and Lemma 7, as $u \rightarrow \infty$, we have

$$
\begin{aligned}
\psi^{*}(u) & =o\left(u^{-(2 p \alpha-\alpha-p) / 2 p}\right)+\frac{1}{\mu^{\alpha} \sqrt{2 \pi}}\left[G\left(\frac{u}{\mu},-1\right)+G\left(\frac{u}{\mu}, 1\right)\right] \\
& \sim \frac{2 u^{-(2 p \alpha-\alpha-1) / 2 p}}{\mu^{(\alpha+1) / 2 p} p \sqrt{2 \pi}} \mathrm{E}\left[\left(\sup _{t \geq 0} \frac{L\left(0, t^{1 / p}\right)}{1+t}\right)^{\alpha}\right] \mathcal{B}\left(\frac{1}{2 p}, \frac{2 p \alpha-\alpha-1}{2 p}\right) .
\end{aligned}
$$

Finally, since $\psi^{*}(u)=2 \psi_{0}(u) / C_{\alpha}$, the desired result follows.

\section{References}

[1] Aaronson, J. (1997). An Introduction to Infinite Ergodic Theory (Math. Surveys Monogr. 50). American Mathematical Society, Providence, RI.

[2] Albeverio, S., Hilbert, A. And Kolokoltsov, V. (1997). Transience of stochastically perturbed classical Hamiltonian systems and random wave operators. Stoch. Stoch. Reports 60, 41-55.

[3] Alparslan, U. T. and Samorodnitsky, G. (2007). Asymptotic analysis of the ruin with stationary stable steps generated by dissipative flows. Scand. Actuarial J. 2007, 180-201.

[4] Alparslan, U. T. and Samorodnitsky, G. (2007). Ruin probability with certain stationary stable claims generated by conservative flows. Adv. Appl. Prob. 39, 360-384.

[5] Asmussen, S. (2000). Ruin Probabilities (Adv. Ser. Statist. Sci. Appl. Prob. 2). World Scientific, River Edge, NJ.

[6] Berman, S. M. (1973/74). Local nondeterminism and local times of Gaussian processes. Indiana Univ. Math. J. 23, 69-94.

[7] Bingham, N. H., Goldie, C. M. And Teugels, J. L. (1989). Regular Variation (Encyclopedia Math. Appl. 27). Cambridge University.

[8] Braverman, M. (2004). Tail probabilities of subadditive functionals on stable processes with continuous and discrete time. Stoch. Process. Appl. 112, 157-183. 
[9] Cohen, S. And Samorodnitsky, G. (2006). Random rewards, fractional Brownian local times and stable selfsimilar processes. Ann. Appl. Prob. 16, 1432-1461.

[10] Embrechts, P. and Maejima, M. (2002). Selfsimilar Processes. Princeton University Press.

[11] Embrechts, P., Klüppelberg, C. And Mikosch, T. (1997). Modelling Extremal Events (Appl. Math. 33). Springer, Berlin.

[12] KAC, M. (1962). Probability theory: Its role and its impact. SIAM Rev. 4, 1-11.

[13] Karatzas, I. And Shreve, S. E. (1991). Brownian Motion and Stochastic Calculus (Graduate Texts Math. 113), 2nd edn. Springer, New York.

[14] Kolokoltsov, V. N. (1997). A note on the long time asymptotics of the Brownian motion with application to the theory of quantum measurement. Potential Anal. 7, 759-764.

[15] Krengel, U. (1985). Ergodic Theorems (de Gruyter Stud. Math. 6). Walter de Gruyter, Berlin.

[16] Lachal, A. (1990). Sur l'intégrale du mouvement brownien. C. R. Acad. Sci. Paris 311, 461-464.

[17] Lachal, A. (1991). Sur le premier instant de passage de l'intégrale du mouvement brownien. Ann. Inst. H. Poincaré Prob. Statist. 27, 385-405.

[18] Lachal, A. (1992). Sur les excursions de l'intégrale du mouvement brownien. C. R. Acad. Sci. Paris 314, 1053-1056.

[19] Lachal, A. (1993). L'intégrale du mouvement brownien. J. Appl. Prob. 30, 17-27.

[20] Lachal, A. (1994). Dernier instant de passage pour l'intégrale du mouvement brownien. Stoch. Process. Appl. 49, 57-64.

[21] Lachal, A. (1995). Sur les temps de passages successifs de l'intégrale du mouvement brownien. C. R. Acad. Sci. Paris 321, 903-908.

[22] Lachal, A. (1996). Sur la distribution de certaines fonctionnelles de l'intégrale du mouvement brownien avec dérives parabolique et cubique. Commun. Pure Appl. Math. 49, 1299-1338.

[23] McKean, H. P., JR. (1963). A winding problem for a resonator driven by a white noise. J. Math. Kyoto Univ. 2, 227-235.

[24] Mikosch, T. and Samorodnitsky, G. (2000). Ruin probability with claims modeled by a stationary ergodic stable process. Ann. Prob. 28, 1814-1851.

[25] Resnick, S. I. (1999). A Probability Path. Birkhäuser, Boston, MA.

[26] Rice, S. O. (1944). Mathematical analysis of random noise. Bell System Tech. J. 23, 282-332.

[27] Rosiński, J. (1995). On the structure of stationary stable processes. Ann. Prob. 23, 1163-1187.

[28] Samorodnitsky, G. (2004). Extreme value theory, ergodic theory and the boundary between short memory and long memory for stationary stable processes. Ann. Prob. 32, 1438-1468.

[29] Samorodnitsky, G. (2004). Maxima of continuous-time stationary stable processes. Adv. Appl. Prob. 36, 805823.

[30] Samorodnitsky, G. And Taqqu, M. S. (1994). Stable Non-Gaussian Random Processes. Chapman and Hall, New York. 